\title{
Endorectal Magnetic Resonance Imaging in Persistent Hemospermia
}

\author{
Adilson Prando \\ Department of Radiology and Diagnostic Imaging, Vera Cruz Hospital, Campinas, Sao Paulo, \\ Brazil
}

\begin{abstract}
Objective: To present the spectrum of abnormalities found at endorectal magnetic resonance imaging (E-MRI), in patients with persistent hemospermia.

Materials and Methods: A review of E-MRI findings observed in 86 patients with persistent hemospermia was performed and results compared with those reported in the literature. Follow-up was possible in 37 of 86 (43\%) patients with hemospermia.

Results: E-MRI showed abnormal findings in 52 of $86(60 \%)$ patients with hemospermia. These findings were: a) hemorrhagic seminal vesicle and ejaculatory duct, isolated $(n=11$ or $21 \%)$ or associated with complicated midline prostatic cyst $(\mathrm{n}=10$ or $19.0 \%)$; b) hemorrhagic chronic seminal vesiculitis, isolated $(\mathrm{n}=14$ or $27 \%$ ) or associated with calculi within dilated ejaculatory ducts $(n=2$ or $4 \%)$; $)$ hemorrhagic seminal vesicle associated with calculi within dilated ejaculatory $\operatorname{duct}(\mathrm{n}=4$ or $7.7 \%)$ or within seminal vesicle $(\mathrm{n}=4$ or $7.7 \%)$; $)$ non-complicated midline prostatic cyst $(\mathrm{n}=6$ or $11.5 \%)$; and e) prostate cancer $(n=1$ or $2 \%)$. Successful treatment was more frequent in patients with chronic inflammatory and/or obstructive abnormalities.

Conclusion: E-MRI should be considered the modality of choice, for the evaluation of patients with persistent hemospermia.
\end{abstract}

Key words: hemospermia; diagnostic imaging; magnetic resonance imaging

Int Braz J Urol. 2008; 34: 171-9

\section{INTRODUCTION}

Hemospermia or hematospermia is not an uncommon clinical urological problem among adult men, but its exact prevalence remains unknown. Hemospermia is prevalent in young males with a mean age of 37 years (1-5). Urogenital inflammation and infection are usually considered the most common cause of hemospermia in this group of patients. In young males often only simple, tailored investigations and appropriate treatment are required. In older patients, above 40 years of age, or those with recurrent hemospermia or associated symptoms, other benign causes and rarely malignancy can be found $(5,6)$.

Imaging evaluation of patients with recurrent hemospermia is usually performed by transrectal ultrasound (TRUS) (7-10). In contrast to TRUS, endorectal magnetic resonance imaging (E-MRI) has the ability to identify hemorrhage within the reproductive structures, but despite its superior diagnostic capability there are only few reports describing its utility in the assessment of persistent hemospermia (10-12). 
Our aim was to illustrate the spectrum of abnormalities found at E-MRI in patients with persistent hemospermia.

\section{MATERIALS AND METHODS}

Between March 2000 and May 2007, 86 consecutive patients with persistent hemospermia of an average duration of 16.7 months (range, 648 months), underwent E- MRI at our institution. Mean patient age was 37 years (range, 25-72 years). Sixty patients $(70 \%)$ were asymptomatic except for hemospermia. One or more associated symptoms, laboratorial or clinical findings were obtained in the remaining 26 patients $(30 \%)$ : frequency or urgency $(\mathrm{n}=10)$, perineal discomfort or pain $(\mathrm{n}=8)$, ejaculatory pain $(n=4)$, arterial hypertension $(n=2)$ and hematuria $(n=2)$. After treatment follow-up was obtained in 37 patients. Conventional MR imaging was performed with a 1.5-T MR imager (Signa; GE Medical Systems, Milwaukee, WI.). Patients were examined by using the body coil for signal acquisition and a combination of a pelvic phased-array coil (GE Medical Systems, Torso PA) with a commercially available balloon-covered endorectal coil (Endo ATD; Medrad, Pittsburgh, PA.), for signal reception. The balloon-covered endorectal coil was inflated with $90 \mathrm{~mL}$ of liquid perfluorocarbon (12). On MR images, the prostate was evaluated with transverse spin-echo T1-weighted MR images by using the following parameters: repetition time $\mathrm{msec} / \mathrm{echo}$ time msec, 575/minimum; section thickness, $3 \mathrm{~mm}$; matrix, 256 x 224; two signals acquired; field of view, $13 \mathrm{~cm}$; intersection gap, $0 \mathrm{~mm}$; bandwidth, 20.83 $\mathrm{kHz}$. Transverse and transverse-oblique T2-weighted images were obtained with the following parameters: $3500 / 130$, section thickness, $3 \mathrm{~mm}$; matrix, $256 \mathrm{x}$ 224, three signals acquired; field of view, $13 \mathrm{~cm}$; intersection gap, $0 \mathrm{~mm}$; bandwidth, $20.83 \mathrm{kHz}$. For the transverse images, phase encoding was in the right-to-left direction. T2-weighted sagittal MR images were obtained with the following parameters: 4000/150; section thickness, $5 \mathrm{~mm}$; matrix, $256 \mathrm{x}$ 192; two signals acquired; field of view, $15 \mathrm{~cm}$; intersection gap, $2 \mathrm{~mm}$; bandwidth, $41.67 \mathrm{kHz}$. For the T2-weighted sagittal MR images, phase encod- ing was in the superior-to-inferior direction. After treatment follow-up was possible in 37 of $86(43 \%)$ patients.

\section{RESULTS}

In patients with hemospermia, E-MRI showed abnormal findings in 52 out of the 86 patients $(60 \%)$. Hemorrhage within the seminal vesicle or the ejaculatory duct was recognized in 45 of 86 patients $(52 \%)$. Blood within seminal vesicle or ejaculatory duct appears as areas of high signal intensity on T1weighted spin-echo images representing the presence of metahemoglobin due to subacute hemorrhage (12).

The imaging criteria used to characterize seminal vesiculitis were: diffuse wall thickening of the seminal vesicle with low T2-weighted signal intensity, loss of convolutions and proteinaceous or hemorrhagic fluid content with variable signal intensity on T1-weighted and T2-weighted images(10). Thus, significant abnormal E-MRI findings observed in this group of patients were: a) hemorrhagic seminal vesicle and ejaculatory duct, isolated $(\mathrm{n}=$ 11 or $21 \%$ ) or associated with complicated midline prostatic cyst ( $n=10$ or $19.0 \%$ ) (Figure- 1$)$; $b)$ hemorrhagic chronic seminal vesiculitis, isolated $(n=14$ or $27 \%$ ) (Figure-2) or associated with calculi within dilated ejaculatory ducts $(\mathrm{n}=2$ or $4 \%)$; $)$ hemorrhagic seminal vesicle associated with calculi within dilated ejaculatory duct $(n=4$ or $7.7 \%)$ or within seminal vesicle ( $n=4$ or $7.7 \%$ ) (Figure-3); d) noncomplicated midline prostatic cyst $(n=6$ or $11.5 \%)$; and e) prostate cancer $(\mathrm{n}=1$ or $2 \%)$, Figure- 4 .

Thirteen patients with hemospermia underwent transurethral endoscopic treatment (unroofing of the midline cysts or ductal obstruction and resection, fulguration, and dilatation of ejaculatory duct obstruction). This approach was successful in 5 patients with dilated hemorrhagic seminal vesicle(s) and ejaculatory duct associated with complicated midline prostatic cyst; in 4 patients with hemorrhagic seminal vesiculitis and calculus within dilated ejaculatory duct and in 1 patient with non-complicated midline prostatic cyst. The same procedure was unsuccessful in 3 patients with non-complicated 

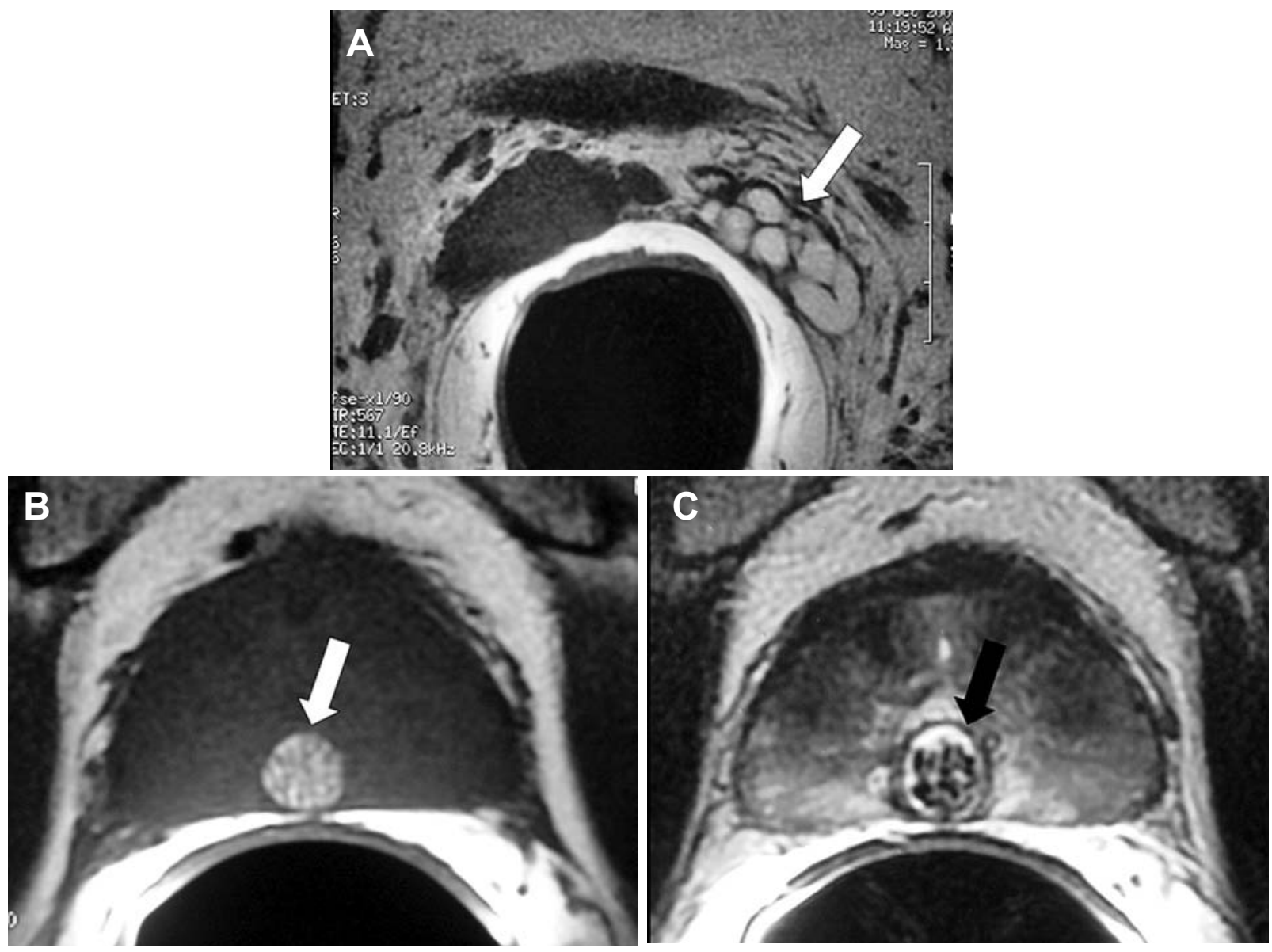

Figure 1 - Hemorrhagic seminal vesicle associated with a complicated midline prostatic cyst (utricular cyst). A 54-year-old man, with history of persistent hemospermia. A) E-MRI, axial plane, T1-weighted image, showing a hemorrhagic normal-walled left seminal vesicle (arrow). Hemorrhage is recognized due the presence of high signal intensity on T1-weighted images. B) and C) E-MRI, axial T1 and T2-weighted images respectively, showing a complicated midline prostatic cyst (arrow) containing blood and several small calculi.

midline prostatic cyst. Hemospermia disappeared completely in 9 out of 12 patients following an E-MRI diagnosis of hemorrhagic chronic seminal vesiculitis and subsequent antimicrobial and or antiinflammatory drugs. Spontaneous elimination of a seminal vesicle calculus was reported by one patient with complete remittance of the hemospermia. Two patients suspected to have prostate cancer due to the presence of focal hypointense area on T2-weighted images, in the peripheral zone of the prostate, were further evaluated with TRUS-guided biopsy guided by magnetic resonance imaging findings (13). This technique allowed the diagnosis of cancer in only one of these patients.

\section{COMMENTS}

Although hemospermia is usually a benign and self-limiting condition, it provokes great concern and anxiety in sexually active patients. Hemospermia may be secondary to inflammation, infection, ductal obstruction or cysts, benign neoplasm, vascular abnormalities, systemic or iatrogenic factors and rarely malignant tumors. History and physical examination are often unrevealing (1). In patients younger than 40 years an infective cause in the urogenital tract is the most common etiological factor (5). Factors that dictate the extent of investigation are patient age, the 


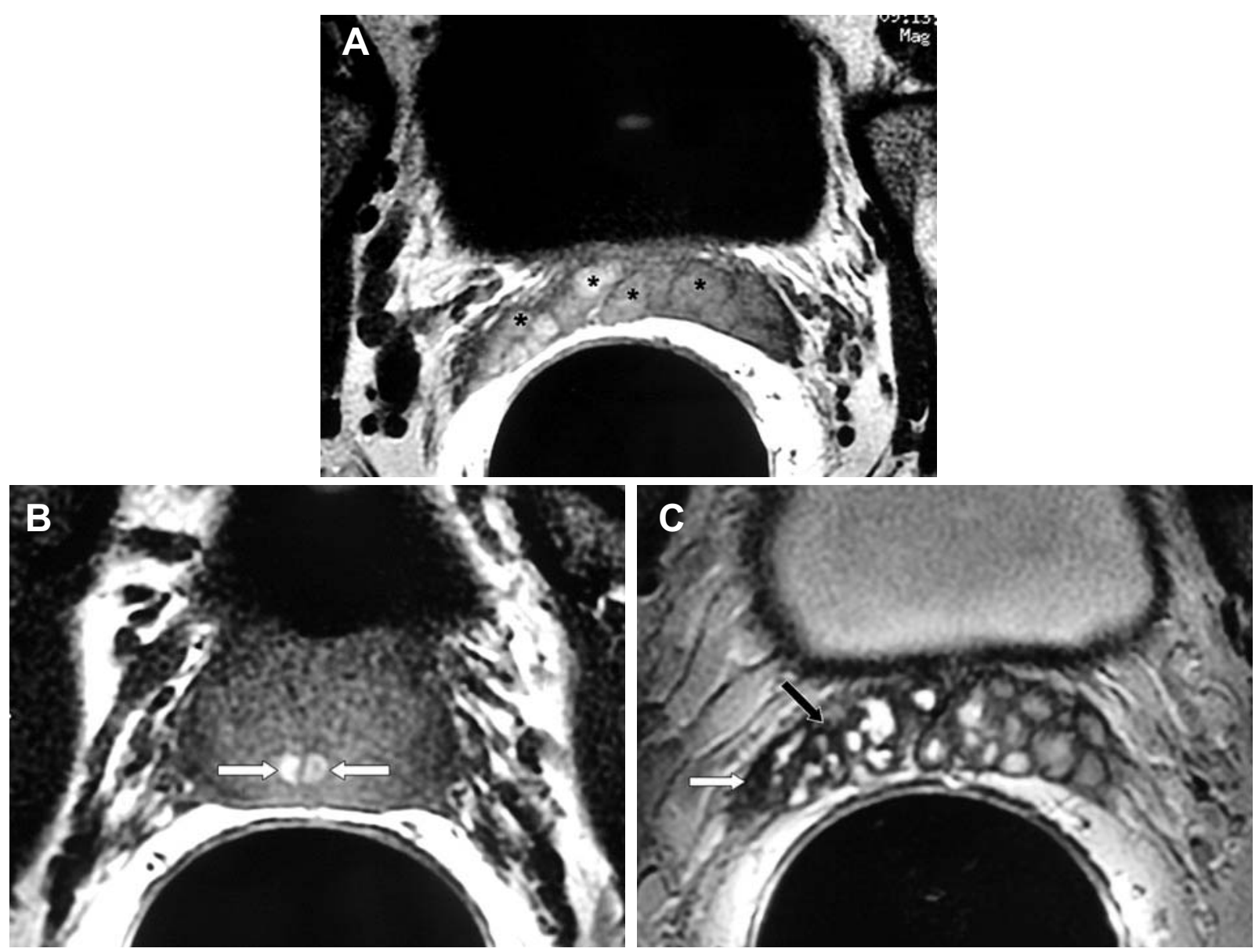

Figure 2-Hemorrhagic chronic seminal vesiculitis. A 62-year-old man, with history of persistent hemospermia and perineal discomfort. A) and B) E-MRI , axial plane, T1-weighted images. Note high-signal intensity hemorrhage in both seminal vesicles (asterisk) and in both ejaculatory ducts (white arrows). C) and D) E-MRI, axial plane, T2-weighted images ,showing imaging features consistent with chronic seminal vesiculitis: diffuse thickening of the of the seminal vesicles with low T2-weighted signal intensity (dark arrow) and loss of convolutions( white arrow).These abnormalities are more evident in the right seminal vesicle which appeared contracted in comparison with the left seminal vesicle.

duration of hemospermia, whether it is persistent and the presence of associated symptoms or signs such as weight loss, local or bony pain, fever, lower urinary tract symptom and hematuria. It is widely accepted that persistent hemospermia or hemospermia with an associated symptom and hemospermia in older patients requires more extensive investigation (1-9).

In our small series of patients, laboratorial or clinical findings were present in 26 out 86 patients(30\%): frequency or urgency $(\mathrm{n}=10)$, perineal discomfort or pain $(n=8)$, ejaculatory pain $(n=4)$, arterial hypertension $(\mathrm{n}=2)$ and hematuria $(\mathrm{n}=2)$. Both patients with hematuria with normal E-MRI findings were submitted to direct rigid and flexible cystoscopy. Papillary urethritis was found in one patient.

TRUS can be considered a safe, noninvasive and relatively inexpensive method, which allows clear images of the reproductive system structures. TRUS has an accurate diagnostic rate of between $74 \%$ and $95 \%$ for the evaluation of hemospermia (5). E-MRI has superior imaging capability since offers higher spatial resolution for the visualization of the whole seminal tract. E-MRI allows the demonstration of normal variations; presence of hemorrhage and evident signs of chronic infection, obstruction 

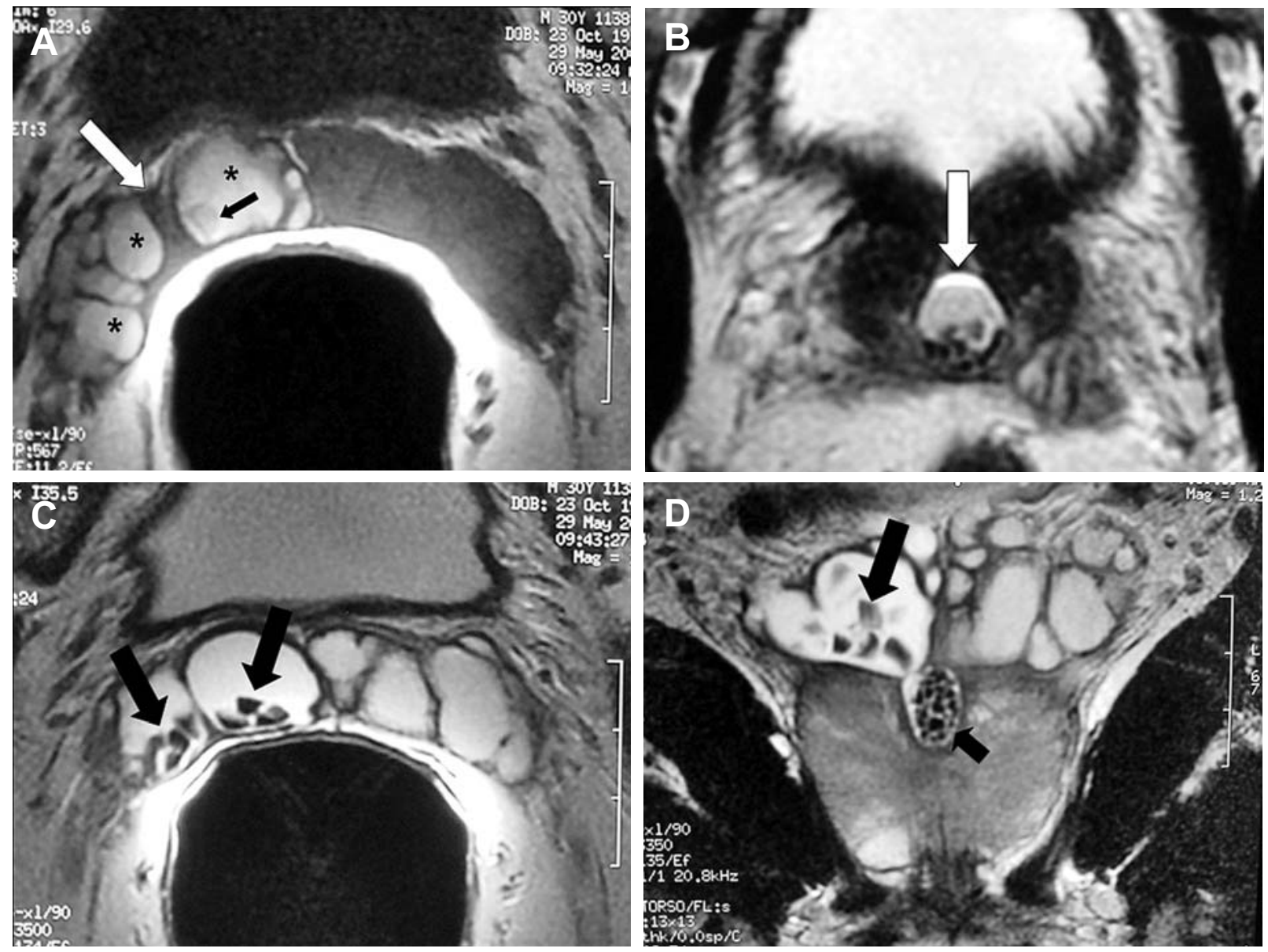

Figure 3 - Hemorrhagic seminal vesicle associated with calculi within the right seminal vesicle and dilated ejaculatory duct. A 30year-old man, with history of persistent hemospermia and ejaculatory pain. A) E-MRI, axial plane, T1-weighted image, showing a hemorrhagic dilated ejaculatory duct (white arrow), containing blood (asterisk) and calculi (black arrow). B) E-MRI, axial,T2-weighted image, better shows the calculi within the dilated ejaculatory duct (arrow). C) and D) E-MRI, axial and coronal T2-weighted images respectively, nicely demonstrates the presence of several stones within the right seminal vesicle (arrows) and within the dilated right ejaculatory duct (small arrow). Note the contiguity of the dilated seminal vesicle with the dilated right ejaculatory duct. This is an essential finding for the differentiation between dilated ejaculatory cyst from midline prostatic cyst.

and malignancies. Contrary to TRUS, MRI has the ability to accurately identify hemorrhage within the seminal tract due to its characteristic signal behavior (high signal intensity on T1-weighted images).

Imaging studies have considered a wide range of etiological factors for hemospermia: prostatic calcification, prostatic hypertrophy, prostatitis, midline prostatic cyst (utricular), midline extra-prostatic cyst, seminal vesicle cyst or calculi, dilatation of the seminal vesicles or the ejaculatory ducts, ejaculatory duct cyst, blood within normal or thick-walled seminal vesicle (seminal vesiculitis) or the ejaculatory duct, seminal vesicle amyloidosis, periprostatic varicosities and prostatic carcinoma (5-11,13-17).

Some of these abnormalities such as prostatic hypertrophy, dilatation of the seminal vesicle(s), prostatic calcification and non-complicated midline prostatic cyst, can be found in asymptomatic patients. Seminal vesicle(s) dilatation for example, has been described as a very common cause of hemospermia $(9,12,14)$ but it is known that various filling states of the seminal vesicles are quite normal. For this reason, we are speculating that perhaps there is a tendency to consider many incidental and common 

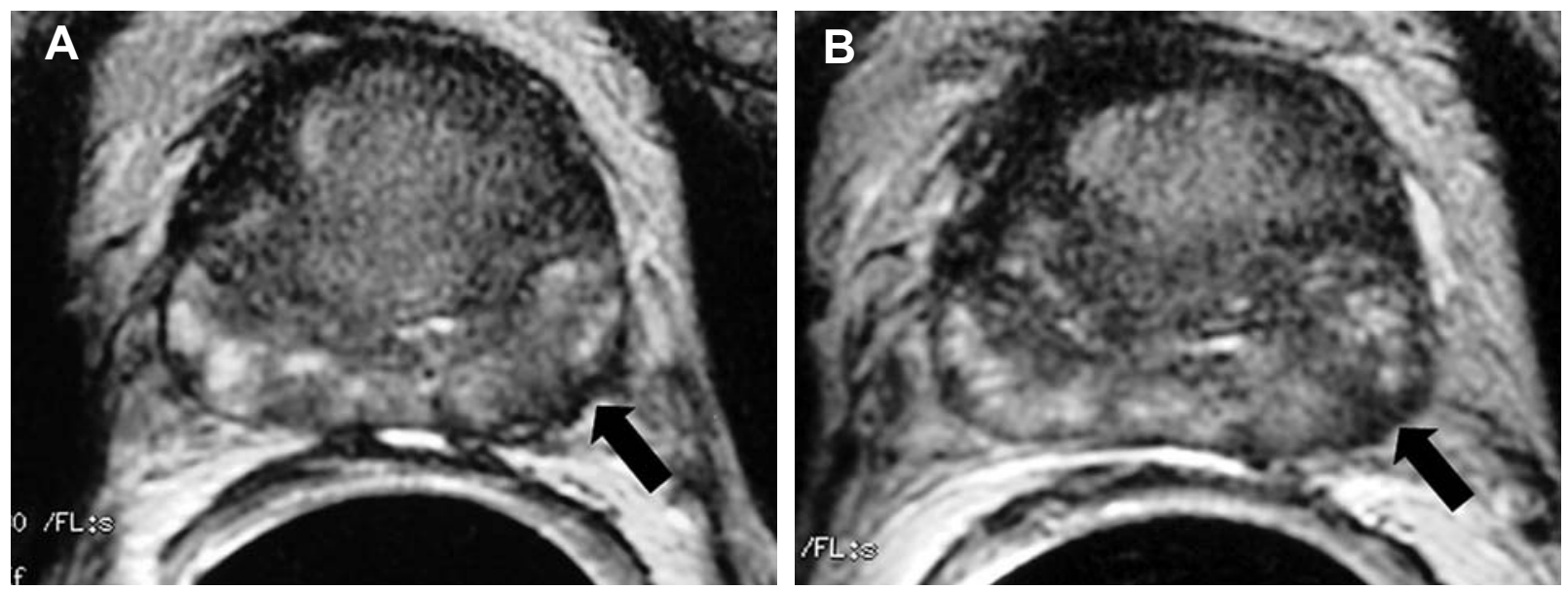

Figure 4-Prostate cancer. A) and B) E-MRI, axial plane, T2-weighted images showing focal area of low signal intensity in the lateral aspect of the left peripheral zone (arrow) associated with irregular thickening of the capsule of the prostate. TRUS-guided biopsy directed by these findings, allowed the diagnosis of prostate cancer, Gleason score (3+4).

urological abnormalities as the etiological factor of hemospermia $(14,18,19)$. This could possibly explain why the success rate of the treatment was variable in our small series of patients. Transurethral endoscopic treatment was more effective in patients with clear obstructive findings and failed in 3 patients with noncomplicated, non obstructive, midline prostatic cyst. This mechanism could also explain why therapy with antimicrobial and or anti-inflammatory drugs was more effective in patients with evident manifestation of seminal vesiculitis and failed in the majority of patients with hemorrhagic seminal vesicle. Although the lack of histological confirmation of chronic seminal vesiculitis (no seminal vesicle biopsy) is a limitation of our study, we may assume that our imaging criteria for chronic seminal vesiculitis is correct since in most of the patients with this MRI findings, hemospermia disappeared after adequate antimicrobial/anti-inflammatory treatment.

In conclusion, E-MRI should be considered the modality of choice for the evaluation of patients with persistent hemospermia. In our series, the most significant E-MRI findings were: hemorrhagic seminal vesicle and ejaculatory duct, isolated or associated with complicated midline prostatic cyst; hemorrhagic chronic seminal vesiculitis, isolated or associated with calculi within dilated ejaculatory ducts, hemorrhagic seminal vesicle associated with calculi within dilated ejaculatory duct or within seminal vesicle, non-complicated midline prostatic cyst and prostate cancer. Successful treatment was, in fact, more frequent in patients with chronic inflammatory and/or obstructive abnormalities.

\section{CONFLICT OF INTEREST}

None declared.

\section{REFERENCES}

1. Munkel witz R, Krasnokutsky S, Lie J, Shah SM, Bayshtok J, Khan SA: Current perspectives on hematospermia: a review. J Androl. 1997; 18: 6-14.

2. Ameur A, Touiti D, Jira H, el Alami M, Boumdin H, Abbar M: Hemospermia: diagnosis and therapeutic aspects. Seven case reports. Ann Urol (Paris). 2002; 36: $74-80$.

3. Fletcher MS, Herzberg Z, Pryor JP: The aetiology and investigation of haemospermia. Br J Urol. 1981; 53: 669-71.

4. Mulhall JP, Albertsen PC: Hemospermia: diagnosis and management. Urology. 1995; 46: 463-7.

5. Ahmad I, Krishna NS: Hemospermia. J Urol. 2007; 177: 1613-8. 
6. Han M, Brannigan RE, Antenor JA, Roehl KA, Catalona WJ: Association of hemospermia with prostate cancer. J Urol. 2004; 172: 2189-92.

7. Worischeck JH, Parra RO: Chronic hematospermia: assessment by transrectal ultrasound. Urology. 1994; 43: 515-20.

8. Etherington RJ, Clements R, Griffiths GJ, Peeling WB: Transrectal ultrasound in the investigation of haemospermia. Clin Radiol. 1990; 41: 175-7.

9. Yagci C, Kupeli S, Tok C, Fitoz S, Baltaci S, Gogus $O$ : Efficacy of transrectal ultrasonography in the evaluation of hematospermia. Clin Imaging. 2004; 28 : 286-90.

10. Torigian DA, Ramchandani P: Hematospermia: imaging findings. Abdom Imaging. 2007; 32: 29-49.

11. Weintraub MP, De Mouy E, Hellstrom WJ: Newer modalities in the diagnosis and treatment of ejaculatory duct obstruction. J Urol. 1993; 150: 1150-4.

12. Lencioni R, Ortori S, Cioni D, Morelli G, Ceretti E, Cosottini M, et al.: Endorectal coil MR imaging findings in hemospermia. MAGMA. 1999; 8: 91-7.

13. Prando A, Kurhanewicz J, Borges AP, Oliveira EM Jr, Figueiredo E: Prostatic biopsy directed with endorectal MR spectroscopic imaging findings in patients with

Correspondence address:

Dr. Adilson Prando

Av. Andrade Neves, 707

Campinas, SP, 13013-161, Brazil

Fax: + $55193231-6629$

E-mail: aprando@mpc.com.br

\section{EDITORIAL COMMENT}

Hemospermia can be considered a challenging situation for both urologists and radiologists, given its relatively high prevalence and poor understanding. Transrectal ultrasound, despite being a good modality for prostate evaluation and guidedintervention, has limited applications for dedicated elevated prostate specific antigen levels and prior negative biopsy findings: early experience. Radiology. 2005; 236: 903-10.

14. Maeda H, Toyooka N, Kinukawa T, Hattori R, Furukawa T: Magnetic resonance images of hematospermia. Urology. 1993; 41: 499-504.

15. Garcia NR, Gozales IF, Mateo CP, Castro GE, Tello AM: Hematospermia y quiste del conducto mülleriano. Arch Esp Urol. 2005; 58: 1061-4.

16. Fuse H, Sumiya H, Ishii H, Shimazaki J: Treatment of hemospermia caused by dilated seminal vesicles by direct drug injection guided by ultrasonography. J Urol. 1988; 140: 991-2.

17. Furuya $\mathrm{S}$, Kato $\mathrm{H}$ : A clinical entity of cystic dilatation of the utricle associated with hemospermia. J Urol. 2005; 174: 1039-42.

18. Ishikawa M, Okabe H, Oya T, Hirano M, Tanaka M, Ono M, et al:: Midline prostatic cysts in healthy men: incidence and transabdominal sonographic findings. AJR Am J Roentgenol. 2003; 181: 1669-72.

19. Curran S, Akin O, Agildere AM, Zhang J, Hricak H, Rademaker J: Endorectal MRI of prostatic and periprostatic cystic lesions and their mimics. AJR Am J Roentgenol. 2007; 188: 1373-9.

Accepted after revision: February 25, 2008

seminal vesicles imaging, especially regarding identification of blood within the ducts (1). The article from Dr. Prando confirms the evolving role of Magnetic Resonance Imaging (MRI) in the evaluation of hemospermia and other seminal vesicles diseases, since it combines high spatial resolution with outstanding 
contrast resolution (the ability to characterize different structures and components, like blood). Endorectal MRI is now considered the modality of choice for local staging of prostate cancer, including seminal vesicles invasion (2). The development and increasing availability of 3 Tesla MR scanners can further improve the application of this imaging modality in the evaluation of the seminal vesicles, since its intrinsic high signal intensity might exempt the need for an endorectal coil (3). Nowadays, regardless these specific technological aspects, we can confidently state that MRI is the imaging modality of choice for evaluation of hemospermia and other seminal vesicles diseases.

\section{REFERENCES}

1. Cho IR, Lee MS, Rha KH, Hong SJ, Park SS, Kim MJ: Magnetic resonance imaging in hemospermia. J Urol. 1997; 157: 258-62.

2. Wang L, Hricak H, Kattan MW, Chen HN, Kuroiwa $\mathrm{K}$, Eisenberg HF, et al.: Prediction of seminal vesicle invasion in prostate cancer: incremental value of adding endorectal MR imaging to the Kattan nomogram. Radiology. 2007; 242: 182-8.

3. Sosna J, Pedrosa I, Dewolf WC, Mahallati H, Lenkinski RE, Rofsky NM: MR imaging of the prostate at 3 Tesla: comparison of an external phased-array coil to imaging with an endorectal coil at 1.5 Tesla. Acad Radiol. 2004; 11: 857-62.

\author{
Dr. Ronaldo Hueb Baroni \\ Radiologist, Body Imaging Department \\ Institute of Radiology, USP and \\ Albert Einstein Israelita Hospital \\ São Paulo, SP, Brazil \\ E-mail:rbaroni@einstein.br
}

\section{EDITORIAL COMMENT}

In his paper published in this issue of the International Brazilian Journal of Urology, Prando presents an overview of abnormalities found at endorectal coil magnetic resonance imaging (MRI) in patients with persistent hemospermia.

The use of MRI instead of imaging modalities such as transrectal ultrasonography or computed tomography seems quite evident. Magnetic resonance imaging allows direct multiplanar image acquisition and offers superb soft tissue contrast, enabling accurate depiction and characterization of soft tissues within the pelvis and facilitating the demonstration of blood products within the male reproductive system. When combined with an endorectal coil, the image resolution can be further increased, providing unsurpassed image detail of the prostate gland, ejaculatory ducts and seminal vesicles. Hence, Prando found abnormalities that were directly related to hemospermia in about $60 \%$ of cases.

On the other hand, uncomplicated hemospermia usually has only minor clinical significance and needs no immediate imaging evaluation, especially in younger patients (less than 40 years of age). However, in cases of persistent or complicated hemospermia, it can be very disquieting for patients and frustrating for urologists to have no information about the location or the etiology of the bleeding. So far, transrectal ultrasonography has been the examination of first choice in these patients. It is a relatively inexpensive and readily available technique that allows the identification of benign prostatic hyperplasia, dilated ejaculatory ducts or seminal vesicles, and obvious lithiasis, cystic lesions, or tumors. On the other hand, transrectal ultrasound cannot directly prove the presence of blood products within the ejaculatory ducts or seminal vesicles and will fail to disclose more subtle abnormalities. Although computed tomography (CT) can readily demonstrate the presence of calcifications and high-density blood within the seminal ductal system, its diagnostic application is hampered because of its low tissue discriminating ability (all structures in and around the prostate and seminal vesicles have about the same density, and also contrast-enhancement is not usually helpful), and because of its radiation exposure, which is not trivial 
in the younger patient group. MRI does not suffer from the abovementioned inconveniences.

We strongly believe, however, that in the majority of patients with hemospermia no immediate imaging evaluation is required. Furthermore, transrectal sonography remains a valid and readily accessible primary technique to disclose more obvious abnormalities of the prostatovesicular complex. However, in complicated or persistent hemospermia, certainly in patients above 40 years of age, MRI may have the potential to disclose more subtle abnormalities that remain obscure on transrectal ultrasonographic examination. Although we are currently not aware of any study having directly compared the diagnostic value of transrectal ultrasound with that of MRI, it is not unreasonable to expect that MRI might become the imaging modality of first choice to evaluate patients with persistent or complicated hemospermia.

Dr. Geert M. Villeirs \& Dr. Willem Oosterlinck Dept of Radiology (GMV) and Urology (WO) Ghent University Hospital Gent, Belgium E-mail: geert.villeirs@ugent.be

Infective cause in the urogenital tract is the most common etiological factor. Dr. Prando confirms findings of inflammatory conditions as a common association with hemospermia and this is demonstrated in recent studies where current laboratory techniques detected a pathogen in $75 \%$ of cases of hemospermia (1).

In summary, current evidence suggests that, for patients with persistent hemospermia, endorectal coil MRI should be performed when TRUS is unsatisfactory or nondiagnostic.

\section{REFERENCE}

1. Bamberger E, Madeb R, Steinberg J, Paz A, Satinger I, Kra-Oz Z, et al.: Detection of sexually transmitted pathogens in patients with hematospermia. Isr Med Assoc J. 2005; 7: 224-7.

Dr. Nelson M. G. Caserta Dept. of Radiology, Unicamp State University of Campinas Campinas, SP, Brazil E-mail:ncaser@mpcnet.com.br 